\title{
Confidence and quality in managing CKD compared with other cardiovascular diseases and diabetes mellitus: a linked study of questionnaire and routine primary care data
}

Mohammad A Tahir ${ }^{1,2^{*}}$, Olga Dmitrieva², Simon de Lusignan², Jeremy van Vlymen², Tom Chan², Ramez Golmohamad', Kevin Harris ${ }^{3}$, Charles Tomson ${ }^{4}$, Nicola Thomas ${ }^{5}$ and Hugh Gallagher ${ }^{1,6}$

\begin{abstract}
Background: Much of chronic disease is managed in primary care and chronic kidney disease (CKD) is a recent addition. We are conducting a cluster randomised study of quality improvement interventions in CKD (QICKD) Clinical Trials Registration: ISRCTN56023731. CKD registers have a lower than expected prevalence and an initial focus group study suggested variable levels of confidence in managing CKD.

Our objective is to compare practitioner confidence and achievement of quality indicators for CKD with hypertension and diabetes.
\end{abstract}

Method: We validated a new questionnaire to test confidence. We compared confidence with achievement of pay-for-performance indicators (P4P) and implementation of evidence-based guidance. We achieved a 74\% (148/ 201) response rate.

Results: $87 \%$ ( $n=128$ ) of respondents are confident in managing hypertension (HT) compared with 59\% ( $n=87)$ in managing $H T$ in CKD (HT+CKD); and with 61\% $(n=90)$ in $H T, C K D$ and diabetes (CKD+HT+DM).

85.2\% (P4P) and $62.5 \%$ (National targets) of patients with hypertension are at target; in patients with HT and CKD $65.1 \%$ and $53.3 \%$; in patients with HT, CKD and DM 67.8\% and 29.6\%.

Confidence in managing proteinuria in CKD is low $(42 \%, n=62) .87 \%$ of respondents knew BP treatment thresholds in CKD, but only 53\% when proteinuria is factored in. Male GPs, younger (<35 yrs), and older ( $>54 \mathrm{yrs}$ ) clinicians are more confident than females and 35 to 54 year olds in managing CKD.

$84 \%$ of patients with hypertension treated with angiotensin modulating drugs achieve achieved P4P targets compared to $67 \%$ of patients with CKD.

Conclusions: Practitioners are less likely to achieve management targets where their confidence is low.

Keywords: Renal Insufficiency, Chronic, Primary Care, Blood pressure, Family Practice, Quality of Healthcare, Proteinuria, Medical Records systems, computerised, Reimbursement, incentives

\section{Background}

The management of cardiovascular disease including hypertension and diabetes is well established in primary care [1-3] and chronic kidney disease (CKD) is a relatively recent addition $[4,5]$. Computerisation of primary

\footnotetext{
*Correspondence: mtahir@nhs.net

'Primary Care Informatics, Division of Public Health Sciences and Education, St George's - University of London, Cranmer Terrace, London SW17 ORE, UK Full list of author information is available at the end of the article
}

care records and explicit national guidance supported by pay for performance (P4P) targets have resulted in a rapid improvement in chronic disease management in primary care [6-9]. In CKD, guidance prioritises the control of systolic blood pressure in patients with proteinuria and or diabetes and recommends the use of angiotensin modulating drugs for achieving control [10-12]. 
CKD is a new priority for primary care and little was known about how to improve quality in CKD [13]. Patients with CKD can be readily identified using a simple formula to estimate glomerular filtration rate (eGFR); a diagnosis is generally made when eGFR is under $60 \mathrm{ml} / \mathrm{min} / 1.73 \mathrm{~m} 2$ on at least two occasions three months apart. People with CKD have a higher mortality and morbidity principally due to cardiovascular disease [4]. Hypertension and diabetes were included in P4P since its inception in 2004. CKD was added in 2006 with performance indicators for: creating a disease register, measuring and controlling $\mathrm{BP}$ and proteinuria, and treating high risk patients with angiotensin converting enzyme inhibitors [14]. However, the prevalence of CKD reported when it was introduced as an indicator in 2006 was only half of that expected from epidemiological studies, suggesting under-ascertainment [15]. We carried out a diagnostic analysis to explore the factors limiting the achievement of quality improvement [16]. This showed that primary care professionals had gaps in their knowledge, highly variable views about this condition and lacked confidence in explaining and managing the condition [17].

We carried out this questionnaire study to assess primary care clinician confidence in the management of CKD and to explore whether confidence may be associated with the quality of care.

\section{Methods}

\section{Subjects}

The subjects of this research are practitioners working in 30 practices drawn from the 127 practices in the Quality Improvement in Chronic Kidney Disease trial (QICKD Clinical Trials Registration: ISRCTN56023731).

At the time of this study we were conducting a cluster randomised trial (CRT) of quality improvement interventions in CKD [18] which includes conducting a process evaluation to explore the reasons why the trial may, or may not succeed [19]. The CRT has three arms: "Audit based education" (feedback of performance compared with peers in an educational context) [20]; "Guidelines and prompts" (postal reminders about management of CKD and copies of NICE guidance [15]); and usual practice. The study has 25 practices per arm to provide sufficient power to detect a change in systolic BP, our primary outcome measure, between the arms. The process evaluation involves six practices which participate in an in-depth evaluation involving focus groups, and an additional 10 practices per arm which form a "Questionnaire group" in order to explore the impact of the interventions on confidence in the management of CKD. The evaluation practices are excluded from the final analysis of the CRT to avoid the possibility of contamination.

\section{Participating practices}

We randomly allocated 10 from each study arm as confidence questionnaire evaluation practices (nested study practices). Although randomly allocated the practices in this nested study differ in age profile from the rest of the QICKD trial and the UK national population. The trial population is very close to the national population in terms of its age-sex distribution [21]. The nested study practices represented $14.9 \%$ of the study sample population $(138,774 / 930,997)$ and their practice populations were 1.5 years younger than those in the nonquestionnaire practices (mean age 37.0 years, SEM 0.046 , compared with 38.5 SEM 0.27; t-test $\mathrm{p}<0.001$ ). There was no significant difference in male: female ratio between questionnaire and non-questionnaire practices (Pearson Chi square $\mathrm{p}=0.660$ ) (Figure 1 ). The crude prevalence of CKD in these practices was $5.4 \%$. The age-standardised prevalence of CKD was higher than that of non-questionnaire practices $(6.3 \%$ vs. $5.3 \%$, OR 1.19: $95 \%$ CI 1.19-1.22, $\mathrm{p}<0.01$ ).

\section{Distribution of the questionnaires and reminders}

We sent a survey questionnaire to 30 practices (201 individual clinicians in total) and collected responses in the three rounds (Figure 2). After the initial postal distribution three rounds of reminders were conducted. Each round involved a telephone reminder to the participant by a researcher to prompt completion by posting a hard copy or to allow the participant to email a scanned copy to a central administrator. Email reminders were also sent at the same time as the phone calls. The telephone reminder was carried out in as unobtrusive a way as possible.

\section{Response rate}

We sent a survey questionnaire to 30 practices (201 individual clinicians in total) and collected responses in the three rounds (Figure 2). At the end 148 questionnaires were returned (74\% response rate) from 93\% (28/ 30) practices.

\section{Developing a confidence questionnaire}

The questionnaire development was carried out in four stages, using an established method [22]. We developed objectives based on our study using knowledge gained from our systematic review [13] and diagnostic analysis [17]. We elected to make comparisons between CKD and other conditions commonly managed in primary care. The objectives of our questionnaire were:

- To compare the confidence of practitioners in controlling systolic BP in patients with CKD with that in patients with hypertension alone. Improved control of systolic BP is the primary outcome measure of the CRT [18]. 

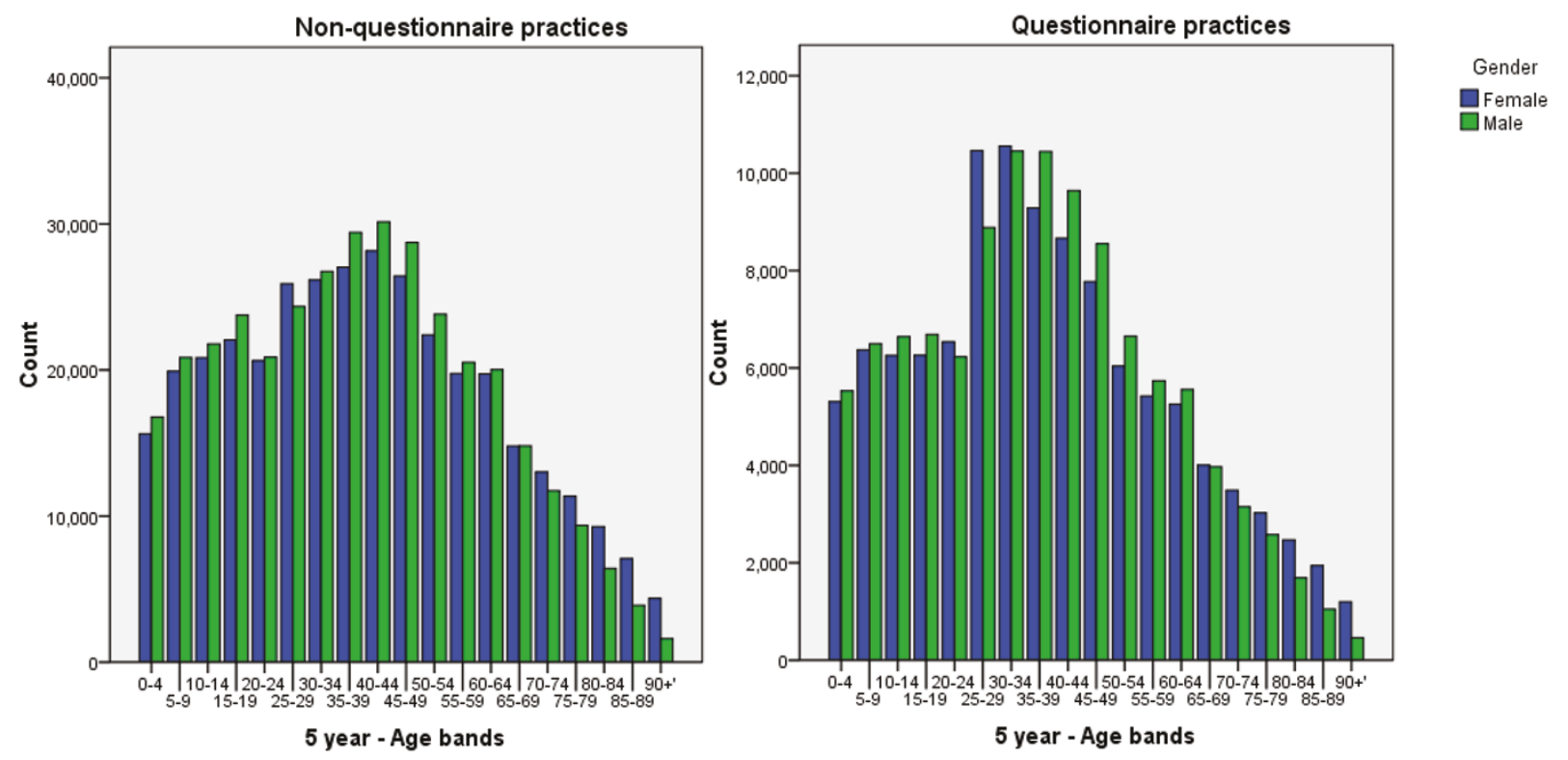

Figure 1 Age-sex profile of non-questionnaire practices (left) with questionnaire practices (right).

- To compare confidence in managing proteinuria in CKD with diabetes. Patients with CKD and proteinuria are at high risk of adverse renal and cardiovascular outcomes [10].

- To compare confidence levels in General Practitioner (GP) partners, salaried GPs and nurses. Our systematic review indicated that successful initiatives in CKD had often been non-doctor led [13].

A draft questionnaire was circulated among the investigators who brainstormed the current issues in CKD management. This draft questionnaire was then piloted on practitioners. This cycle was repeated on three occasions. We conducted three focus groups $(\mathrm{n}=7,6,8)$ to refine the questionnaire using groups of general practitioners, practice nurses and medical students to validate the questionnaire. These focus groups were facilitated by the principal author to this paper.

The validation process involved the practitioners completing the questionnaire and then marking a colleague's questionnaire while discussing the responses and highlighting potential ambiguities. The focus groups were reviewed and evaluated using a field notebook and transcriptions of tape recordings. A smaller group of GPs were contacted via email $(n=3)$ to ensure adequate validity.

We elected to include a small number of key competence measures as we felt that we could not interpret confidence entirely isolated from competence. These focussed on knowledge of BP targets for treatment, intervention levels of proteinuria and criteria for referral. The questionnaire consisted of 24 confidence questions rated 1 to 5 (Additional File 1) and 6 knowledge questions. The confidence questions used a Likert scale from 1 to 5 , where 1 is "Not confident at all" and 5 is "Very confident."

\section{Collecting routine clinical data}

We collected anonymised routine clinical data from the computerised medical record systems of the questionnaire practices to enable us to compare confidence with achievement. We extracted the key variables associated with the questionnaire or required to make comparisons: practice identifier, age and gender, BP, significant proteinuria, CKD (strictly applying a standard for chronicity), diabetes (again using strict diagnostic criteria) [23] or hypertension. Data were processed using an established method [24] and analysed in SPSS (Statistical Package for Social Sciences, Version 16).

\section{Analysis plan}

Our analysis had four stages:

1. Comparing confidence levels and knowledge for different age, gender and type of practitioner, and comparing confidence in management of CKD with that of hypertension and diabetes. We judge a person who scored 4 or 5 on the questionnaire to be "confident" and one scoring 1, 2 or 3 to be "not-confident".

2. Establishing BP control and proportion at target for people with CKD, with and without significant proteinuria, diabetes and hypertension. 


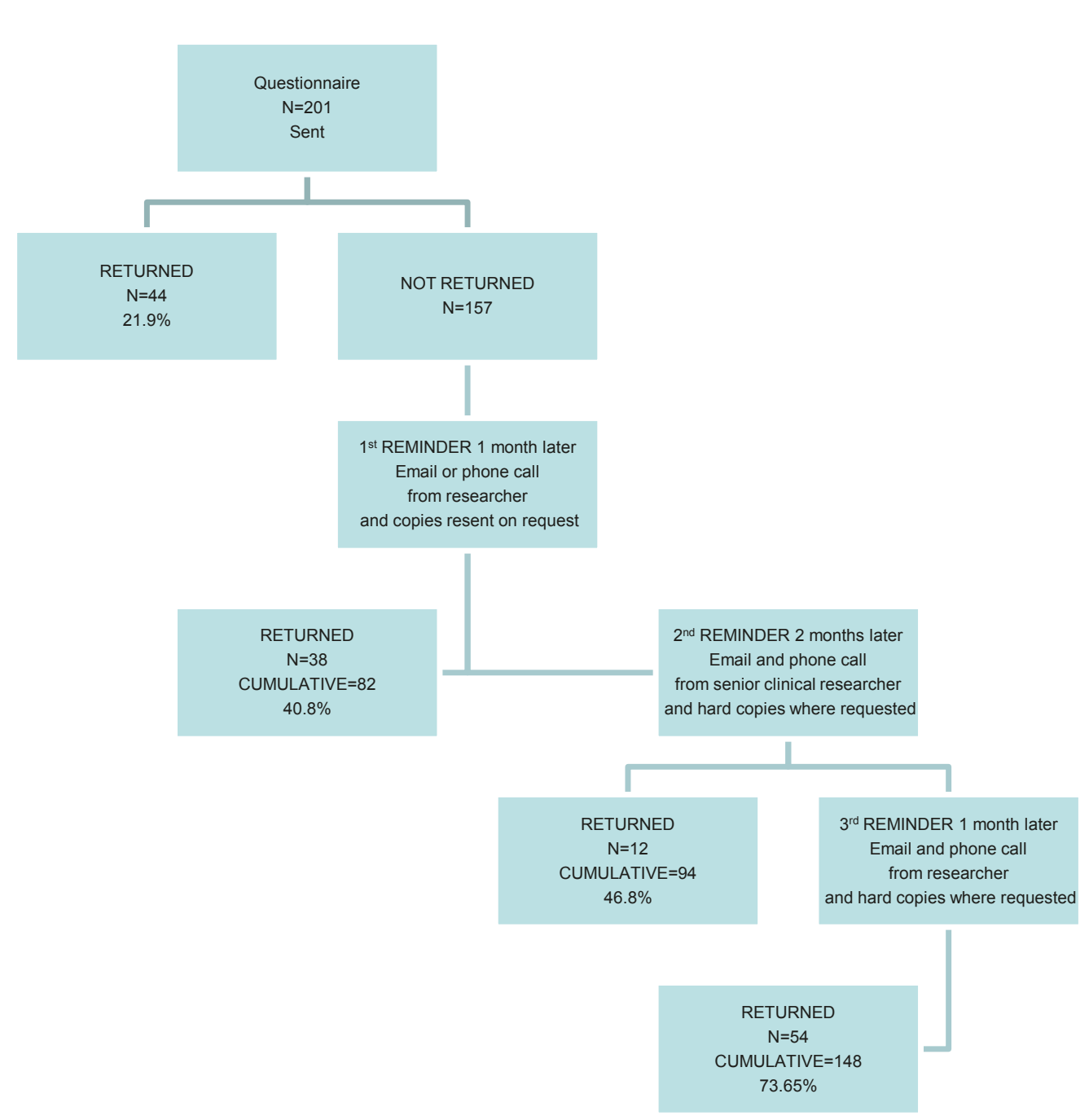

Figure $\mathbf{2}$ The scheme of distribution and collection of questionnaires.

3. A comparison of level of confidence and achievement of the blood pressure targets set out in national evidence-based guidance [10] and P4P indicators [11]. To do this we calculated a mean confidence score based on the responses from each practice and compared this with the achievement of P4P indicators by that practice.

4. Investigating variation in practice response

\section{Statistical analysis}

We used a range of descriptive and inferential statistical procedures to compare differences in the samples and outcomes. Where appropriate, we report the percentages and $95 \%$ confidence intervals (CI) to allow comparison between subgroups. For categorical variables, we used the Pearson Chi square test to detect differences between sub-groups or associations between variables. We performed this analysis in SPSS version 16.

\section{Ethical Considerations}

The study has been approved by the Oxford Research Ethics committee and is a registered clinical trial (ISRCTN56023731).

\section{Results}

The final response rate was $74 \%$. After three rounds of reminders we received 148 questionnaires from 201 primary care professionals from $93 \%(28 / 30)$ of the practices.

We found that most participants, $86.5 \%(\mathrm{n}=128$; $95 \%$ CI: $80.1 \%$ to $91.1 \%$ ) in our study are confident in managing hypertension whereas only $58.8 \%(n=87$; CI: $50.7 \%$ to $66.4 \%$ ) are confident in the management of hypertension in patients with CKD, and 60.8\% ( $\mathrm{n}=90$; CI: $52.7 \%$ to $68.3 \%$ ) are confident in managing hypertension in patients with CKD and diabetes (DM). Just 
under half of the respondents, $49.3 \%(\mathrm{n}=73$; CI: $41.4 \%$ to $57.3 \%$ ) are confident in achieving lowered blood pressure in patients with CKD (Figure 3).

Importantly, confidence is especially low in the management of patients with CKD and significant proteinuria, who are at higher risk of adverse renal and cardiovascular outcomes. $42.6 \%(\mathrm{n}=63$; CI: $34.9 \%$ to $50.6 \%$ ) of respondents lack confidence with identifying significant proteinuria in patients with CKD and only $41.9 \%$ ( $n=62$; CI: $34.2 \%$ to $49.9 \%$ ) are familiar with using urine protein results to manage CKD. Practitioners are more confident in identifying significant proteinuria in patients with DM, $64.9 \%(\mathrm{n}=96$; CI: $56.9 \%$ to $72.1 \%$ ), and more confident in using urine protein results to manage DM, $47.3 \%(\mathrm{n}=70$; CI: $39.4 \%$ to $55.3 \%)$ than in patients with CKD.

About a third of respondents lack confidence with using ACE-I and/or ARB in patients with CKD, 33.8\% $(n=50$; CI: $26.7 \%$ to $41.7 \%) .66 .9 \%(n=99$; CI: $59.0 \%$ to $74.0 \%)$ of respondents lack confidence with adding a loop diuretic drug (e.g. furosemide) to patients with CKD already on maximum dose of an ACE-I and/or ARB. 35.8\% ( $n=53$; CI: $28.5 \%$ to $43.8 \%$ ) of respondents lack confidence in using anti-hypertensives that are not in the angiotensin modulating category in patients with CKD (Figure 4).

More male GPs reported feeling confident in the overall management of CKD than female GPs, but the difference is not statistically significant $(61.5 \%$ vs. $50.9 \%$, Pearson Chi Square $\mathrm{p}=0.242$ ). Male GPs (salaried GPs and partners) are more confident in managing hypertension in patients with CKD $(76.9 \%$ vs. $54.5 \%, \mathrm{p}=0.01)$ and in managing hypertension in patients with CKD and DM $(73.8 \%$ vs. $56.4 \%, \mathrm{p}<0.05)$ than female GPs. Also male GPs are more confident with using an ACE-I and/or ARB or other anti-hypertensive(s) in patients with CKD $(86.2 \%$ and $84.6 \%$ vs. $65.5 \%$ and $63.6 \%$, p < 0.01 ) compared with their female counterparts.

When it comes to adding a loop diuretic drug (e.g. furosemide) to patients with CKD already on maximum

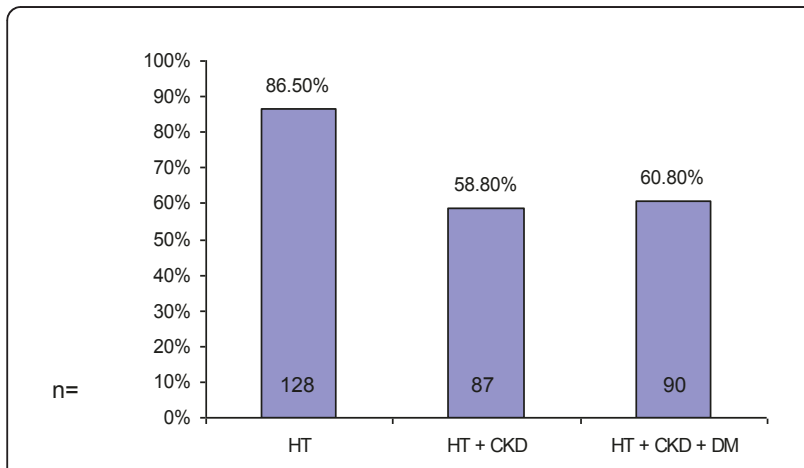

Figure 3 Confidence in managing hypertension.

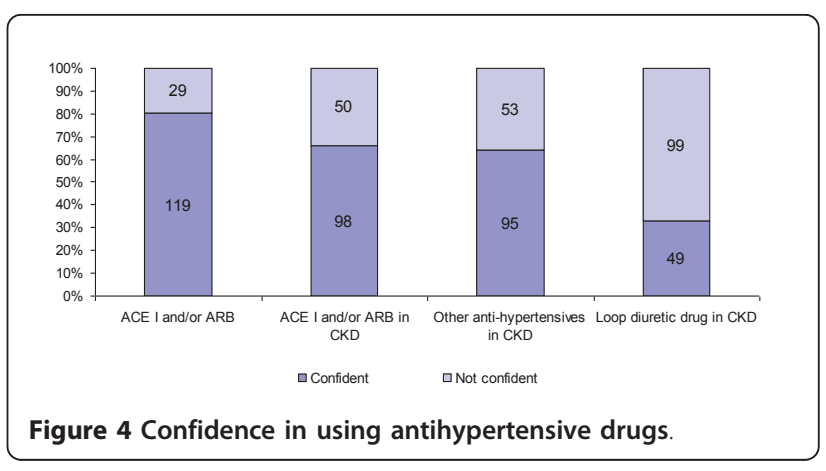

dose of an ACE I and/or ARB, male GPs are approximately twice as confident as female GPs $(50.8 \%$ vs. $25.5 \%, \mathrm{p}=0.005)$.

Confidence in the management of patients with CKD was ranked by respondents' age (Table 1). Younger clinicians $(<35$ year old) are more confident in managing hypertension than the $35-54$ year old group (100.0\% vs. $80.6 \%, \mathrm{p}=0.023)$. At the same time, more of the younger $(<35$ year old) and more of the older ( 55 and older) practitioners are confident in the management of hypertension in patients with CKD than the 35-54 year old group $(69.6 \%, 77.4 \%$ vs. $50.0 \%, \mathrm{p}=0.014) .73 .9 \%$ of the $<35$ years old group but only $39.4 \%$ of $35-54$ years olds are confident in achieving lowered blood pressure in patients with CKD ( $\mathrm{p}=0.004)$.

The younger group are significantly more confident than the 35-54 year old group in interpreting eGFR to stage CKD (87\% vs. $61.7 \%, \mathrm{p}=0.02)$ and monitoring eGFR in patients with CKD (87\% vs. $61.7 \%, \mathrm{p}=0.033)$, and in monitoring eGFR in CKD patient with DM $(69.6 \%$ vs. $51.1 \%$, p $=0.01$ ). Only about half of respondents in each group felt confident in using urine protein results to manage DM $(47 \%, \mathrm{p}=0.034)$ and in CKD $(41.9 \%, \mathrm{p}=0.3)$.

The $>54$ year old group have the highest confidence, compared to the 35-54 year old and $<35$ year old groups, in using referral guidelines to refer appropriate patients with either both DM and CKD (83.9\% vs. 52.2\% vs. $54.3 \% \mathrm{p}=0.01)$, or CKD alone $(80.6 \%$ vs. $56.5 \%$ vs. $45.7 \% \mathrm{p}=0.03)$ to secondary care.

$17.4 \%$ and $41.5 \%$ of respondents in the $<35$ year old group and the 35-54 year old group respectively are not confident with using ACE-I and/or ARB in patients with CKD. This difference was statistically significant $(\mathrm{p}=$ $0.03)$.

$86.5 \%(\mathrm{n}=128)$ of practitioners were able to correctly identify the P4P thresholds for blood pressure control in patients with CKD without proteinuria compared with $53.4 \%(\mathrm{n}=79)$ of respondents when proteinuria is factored in.

Next we evaluated clinical outcomes in the management of blood pressure. The mean systolic BP in the 
Table 1 Confidence in managing CKD and DM in different age groups

\begin{tabular}{|c|c|c|c|c|c|c|c|}
\hline \multirow[t]{2}{*}{ Confidence } & \multicolumn{2}{|r|}{$0-34$} & \multicolumn{2}{|c|}{$35-54$} & \multicolumn{2}{|c|}{$55+$} & \multirow[t]{2}{*}{$p$} \\
\hline & $\mathrm{N}$ & $\%$ & $\mathrm{~N}$ & $\%$ & $\mathrm{~N}$ & $\%$ & \\
\hline Managing hypertension & 23 & $100.0 \%$ & 75 & $80.6 \%$ & 29 & $93.5 \%$ & 0.02 \\
\hline Managing hypertension in patients with CKD & 16 & $69.6 \%$ & 47 & $50.0 \%$ & 24 & $77.4 \%$ & 0.01 \\
\hline Managing hypertension in patients with CKD with DM & 15 & $65.2 \%$ & 52 & $55.3 \%$ & 23 & $74.2 \%$ & 0.16 \\
\hline Achieving lowered blood pressure in patients with CKD & 17 & $73.9 \%$ & 37 & $39.4 \%$ & 19 & $61.3 \%$ & 0.00 \\
\hline Interpreting eGFR to stage CKD & 20 & $87.0 \%$ & 58 & $61.7 \%$ & 25 & $80.6 \%$ & 0.02 \\
\hline Monitoring eGFR in patients with CKD & 20 & $87.0 \%$ & 58 & $61.7 \%$ & 24 & $77.4 \%$ & 0.03 \\
\hline Monitoring eGFR in CKD patient with DM & 16 & $69.6 \%$ & 48 & $51.1 \%$ & 25 & $80.6 \%$ & 0.01 \\
\hline Using urine protein results to manage DM & 15 & $65.2 \%$ & 37 & $39.4 \%$ & 18 & $58.1 \%$ & 0.03 \\
\hline Using urine protein results to manage CKD & 12 & $52.2 \%$ & 35 & $37.2 \%$ & 15 & $48.4 \%$ & 0.31 \\
\hline Using ACE inhibitors and/or ARB & 22 & $95.7 \%$ & 71 & $75.5 \%$ & 26 & $83.9 \%$ & 0.08 \\
\hline Using ACE inhibitors and/or ARB in patients with CKD & 19 & $82.6 \%$ & 55 & $58.5 \%$ & 24 & $77.4 \%$ & 0.03 \\
\hline Using other anti-hypertensives in patients with CKD & 18 & $78.3 \%$ & 54 & $57.4 \%$ & 23 & $74.2 \%$ & 0.08 \\
\hline $\begin{array}{l}\text { Adding a loop diuretic drug (e.g. furosemide) to patients with CKD already on maximum dose of an ACE I } \\
\text { and/or ARB }\end{array}$ & 7 & $30.4 \%$ & 26 & $27.7 \%$ & 16 & $51.6 \%$ & 0.05 \\
\hline Using referral guidelines to refer appropriate patients with DM to secondary care & 12 & $52.2 \%$ & 51 & $54.3 \%$ & 26 & $83.9 \%$ & 0.01 \\
\hline Using referral guidelines to refer appropriate patients with CKD to secondary care & 13 & $56.5 \%$ & 43 & $45.7 \%$ & 25 & $80.6 \%$ & 0.00 \\
\hline Overall management of patients with DM & 15 & $65.2 \%$ & 54 & $57.4 \%$ & 26 & $83.9 \%$ & 0.03 \\
\hline Overall management of patients with CKD & 9 & $39.1 \%$ & 44 & $46.8 \%$ & 22 & $71.0 \%$ & 0.03 \\
\hline
\end{tabular}

study population as a whole was $125 \mathrm{mmHg}$ and in the population without any cardiovascular (CVS) co-morbidity was $122 \mathrm{mmHg}$.

Analysis of overall practice performance in BP management revealed poor $\mathrm{BP}$ control in patients with CKD and low numbers of patients reaching BP goals (both NICE targets and P4P payment thresholds, Table 2). Only half of patients (53.3\%) with CKD and hypertension have achieved the NICE target for blood pressure management whereas this number was $62.5 \%$ for people with hypertension without CKD. In patients with CKD and diabetes this figure drops to $29.6 \%$. Currently P4P thresholds for BP are higher than NICE. $67.80 \%$ of patients with CKD and DM meet P4P thresholds for BP management.
Patients with CKD and both proteinuria and diabetes are a high risk group in whom lowering systolic BP is particularly important. There appears to be a lack of confidence in treating these patients and this is reinforced by a low achievement of BP targets, with only $31.3 \%$ patients meeting at goal of $130 \mathrm{mmHg}$ as recommended by NICE.

$70 \%$ of patients with $\mathrm{HT}$ received angiotensin modulating drugs for blood pressure treatment (mean BP achieved $138.6 \mathrm{mmHg}$ ). $61.4 \%$ of these patients meet NICE targets and $84.2 \%$ meet P4P thresholds for BP control.

79\% of CKD patients (mean 136.3) received ACE-I or ARB. However, in the same group, only $48.8 \%$ (NICE) and $67.0 \%(\mathrm{P} 4 \mathrm{P})$ of patients are at target for BP control.

Table 2 Confidence and achievements in BP management in patients with CKD and DM

\begin{tabular}{|c|c|c|c|c|c|c|}
\hline \multirow[b]{2}{*}{ Managing $\mathrm{HT}$} & \multirow{2}{*}{$\begin{array}{c}\text { Confidence \% } \\
86.5 \%\end{array}$} & \multirow{2}{*}{$\begin{array}{c}\text { BP Mean } \\
138.07\end{array}$} & \multicolumn{2}{|c|}{ Patients at NICE target } & \multicolumn{2}{|c|}{ Patients at P4P target } \\
\hline & & & 8701 & $62.5 \%$ & 11853 & $85.2 \%$ \\
\hline Managing $\mathrm{HT}$ in patients with $\mathrm{CKD}$ & $58.8 \%$ & 137.43 & 2575 & $53.3 \%$ & 3141 & $65.1 \%$ \\
\hline Managing $H T$ in CKD with $\mathrm{DM}$ & $60.8 \%$ & 136.26 & 420 & $29.6 \%$ & 963 & $67.8 \%$ \\
\hline Urine protein results to manage DM & $47.3 \%$ & 135.38 & 193 & $32.5 \%$ & 467 & $78.6 \%$ \\
\hline Urine protein results to manage CKD & $41.9 \%$ & 140.96 & 5 & $21.7 \%$ & 16 & $69.6 \%$ \\
\hline ACE-I and/or ARB in HT only & $80.4 \%$ & 138.6 & 5330 & $61.4 \%$ & 7310 & $84.2 \%$ \\
\hline ACE-I and/or ARB in CKD & $66.2 \%$ & 136.3 & 2796 & $48.8 \%$ & 3841 & $67.0 \%$ \\
\hline Assessing CVD risk scores in DM & $71.6 \%$ & 128.8 & 456 & $71.0 \%$ & 516 & $80.4 \%$ \\
\hline Assessing CVD risk scores in CKD & $63.5 \%$ & 129.7 & 232 & $73.9 \%$ & 277 & $88.2 \%$ \\
\hline Overall management of DM & $64.2 \%$ & 133.2 & 3556 & $46.4 \%$ & 6377 & $83.2 \%$ \\
\hline Overall management of CKD & $50.7 \%$ & 133.9 & 6262 & $56.4 \%$ & 7932 & $71.4 \%$ \\
\hline
\end{tabular}


Practitioner confidence is also low so far as treatment of these conditions are concerned.

Practitioner confidence is lowest in managing the following groups; patient with CKD, with DM and proteinuria, and with CKD and proteinuria (58.1\%, 47.3\% and $41.9 \%$ respectively). CKD combined with DM or HT or both produce higher confidence values than that of CKD alone.

We compared individual practice responses to see if outliers or practice variation might account for a difference in confidence levels. We found no difference related to list size and similar results for the different patient groups (Figure 5).

\section{Discussion}

\section{Principal findings}

Practitioners are less confident in managing CKD than hypertension or diabetes. There is also a lack of confidence in managing proteinuria, combined with a knowledge gap in interpreting proteinuria test results.

Within the respondent group, salaried doctors, males, older and younger doctors and GPs compared to nurses were more confident in managing CKD. Clinicians are least confident in managing individuals with CKD who are at highest risk, i.e. those with proteinuria. Confidence is lower in managing patients with proteinuria and CKD than those patients with proteinuria and diabetes.
The quality of care in CKD, measured by ascertainment of standards in national guidance and for P4P is lower where confidence is low. This is particularly apparent in CKD and in people with CKD and diabetes.

Nearly all (> 80\%) of people with hypertension taking an angiotensin modulating drug achieve national quality targets compared with two-thirds of people with CKD.

\section{Implications for practice}

Low practitioner confidence and knowledge gaps may be reversed by educational interventions. The QICKD study, using information (guidelines and prompts) and education (audit based education) will inform whether these interventions change confidence and ultimately the quality of care. These interventions were chosen as they represented two common initiatives used in primary care to improve care and thought to prompt change in practice.

Interventions may need to be tailored to meet the needs of different practitioner groups, as there are marked differences between the groups of practitioners. Nurses in particular should have specific training as this clinician group deliver much of the chronic disease management in primary care and increasingly chronic kidney disease management. One consequence of P4P may be that practices improve quality up to the standard required to achieve the indicator. The highest risk patients, who may also be the most difficult to

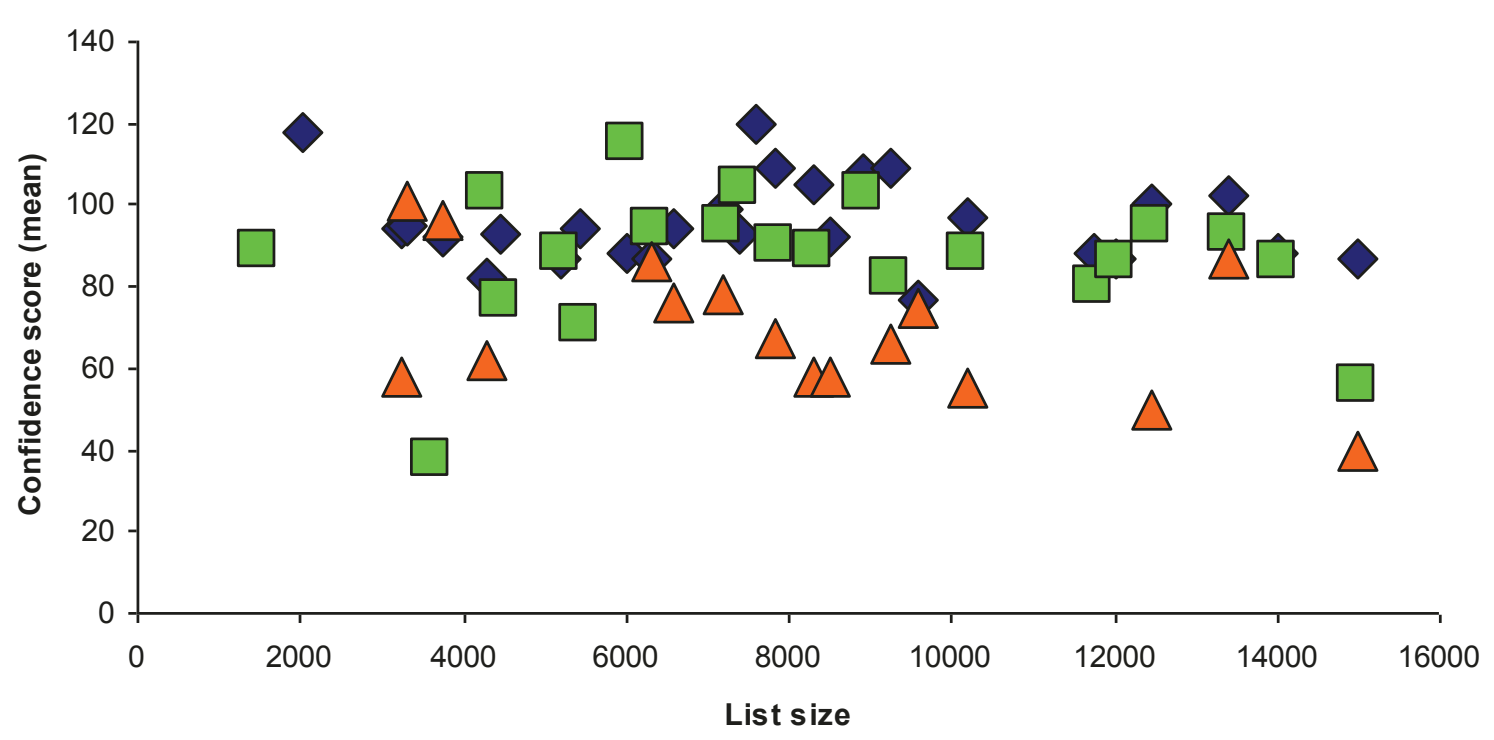

Salaried GP $\quad \square$ Partner $\quad \Delta$ Nurse

Figure 5 Salaried GP, GP Partner and Practice Nurse scores across practice list size. 
manage, may not be optimally treated. Incentives could be tailored to reward achievement of NICE thresholds for the highest risk patients, for example the use of Local Enhanced Services in Primary Care Trusts in England.

\section{Comparison with the literature}

Other studies have shown a knowledge gap in CKD management $[25,26]$ reinforcing the findings of our own diagnostic analysis [17]. There are some pointers from educational research and from other disease areas that level of knowledge $[27,28]$ and confidence is associated with improved practice [29-32]; however most of these articles are descriptive rather than conducted in trials.

It may be premature to presume that the patterns of practitioner confidence described in our study are generaliseable. One study of knowledge of CKD showed it declined with increasing practitioner age [25]. Another, in the field of dementia [31] suggested younger and female GPs had greater knowledge and awareness; whereas the exploration of discussing sexual difficulties showed no gender difference [32].

\section{Limitation of the method}

There may be a number of sources of bias in this investigation. Firstly, the practices participating in the QICKD study are volunteer practices who may not be representative of the wider population. Secondly, we have no information about the non-responders.

We can only look at comparing individual practitioners' responses with practice level outcome data. This is because most practices allow patients to see a number of doctors.

\section{Call for future research}

We need to determine the cost effectiveness of any educational intervention updating economic modelling based on previous guidance [33]. Further studies which provide educational or other interventions to people who lack confidence in managing a condition should be conducted to determine whether this improves the quality of care.

\section{Conclusion}

Practitioners are less confident in managing CKD than hypertension or diabetes and are less likely to achieve BP targets where confidence is low. Improving knowledge and confidence may provide the key to improving the quality of CKD management in primary care, especially for higher risk patients with diabetes and with proteinuria.

\section{Additional material}

Additional file 1: Questionnaire: How confident are General Practitioners \& the Primary care team in managing Chronic Kidney Disease (CKD).

\section{Acknowledgements}

The participating practices and the members of QICKD study team. Funding is primarily through the Health Foundation with additional funding from the Edith Murphy Foundation.

\section{Author details}

${ }^{1}$ Primary Care Informatics, Division of Public Health Sciences and Education, St George's - University of London, Cranmer Terrace, London SW17 ORE, UK ${ }^{2}$ Department of Health Care Management and Policy, University of Surrey, Guildford, UK, Surrey GU2 7XH. ${ }^{3}$ University Hospitals of Leicester NHS Trust, John Walls Renal Unit, Leicester General Hospital, Leicester LE5 4PW UK, UK ${ }^{4}$ Department of Renal Medicine, Southmead Hospital, Bristol BS10 5NB, UK. ${ }^{5}$ School of Community and Health Sciences, City University London, 20 Bartholomew Close, London EC1A 7QN, UK. ${ }^{6}$ SW Thames Renal \& Transplantation Unit, St Helier Hospital, Wrythe Lane Carshalton, Surrey SM5 1AA UK, UK.

\section{Authors' contributions}

$A T^{1,2}$ : Developed and validated confidence questionnaire, contributed to analysis of data, contributed to each version of the paper. $\mathrm{OD}^{2}$ : Conducted major role in analysis of data, created $1^{\text {st }}$ draft and contributed to each version. SdeL ${ }^{2}$ : Developed the idea of linking confidence to live practice data, contributed to analysis of data, major part in drafting and redrafting paper, PI of QICKD study. JVV² : Cleaned and analysed raw practice data, contributed to final version of paper. $\mathrm{TC}^{2}$ : Contributing to developing questionnaire, analysis of data, contributed to final version of paper. $\mathrm{RG}^{1}$ : Major Input to early analysis of data, contributed to final version of paper. $\mathrm{KH}^{3}$ : Contributed to Research Idea, contributed to final version of paper. $\mathrm{CT}^{4}$ : Contributed to Research Idea, contributed to final version of paper. $\mathrm{NT}^{5}$ : Contributed to Research Idea, contributed to final version of paper. $\mathrm{HG}^{1,6}$ : Contributed to Research Idea, contributed to final version of paper. All authors declare that the answers to the questions on your competing interest form are all "No". All authors have read and approved the final manuscript.

\section{Competing interests}

$\mathrm{AT}^{1,2}$ Nil declared

$\mathrm{OD}^{2}$ Nil declared

SdeL $^{2}$ SdeL is the GP expert advisor for the Quality and Outcomes Framework (QOF - a pay-for-performance (P4P) scheme) with the role of developing a CKD Indicator. This was done on behalf of NICE (National Institute for Health and Clinical Excellence). SdeL has received funding for research staff from Roche for the data analysis which formed part of the NEOERICA study. He has received sponsorship from Pfizer to speak at two cardiovascular meetings in 2008; received an honorarium for writing a magazine article (Prescriber) jointly with $\mathrm{HG}$.

$\mathrm{JVV}^{2}$ For two years JVV's salary was part-funded by the NEOERICA study $\mathrm{TC}^{2}$ Nil declared

$\mathrm{RG}^{1}$ Nil declared

$\mathrm{KH}^{3}$ Funding: Grants Pfizer International Doxazosin Award 2003: The role of alpha blockade on matrix synthesis by mesangial cells - £10,000; Pfizer award 2004: To investigate the effect of atorvastatin on renal reperfusion injury - $£ 12,000$; Health Foundation 2007-2010: Quality Improvement in CKD: a challenge for primary care - £695,000; Edith Murphy Foundation 20072010: Quality Improvement in CKD due to diabetes - $£ 450,000$; LNR CLAHRC 2008-2014: Prevention of Chronic Disease and its Associated Co-Morbidity theme - $c £ 4$ million out of $c £ 20$ million total. Funding: others in last 5 years (travel support \& ad hoc honararia) Roche, Ortho Biotech, Amgen, Baxter, Boehringer. Other: Advisory Board Membership Roche, Genzyme, Shire, Baxter, Novartis. 
$\mathrm{CT}^{4} \mathrm{Dr}$ Tomson was a member of advisory board of Genzyme UK between April 2002 and May2004. He had received honoraria from Janssen-Cilag, MSD, Bayer, Novartis, Baxter Healthcare, Amgen, Genzyme, AstraZeneca, Pfizer, Roche. He is a current member of International Steering Committee of the Study of Heart and Renal Protection.

$N T^{5}$ Funding: Grants Hospital Savings Association - $£ 5,000$; Kidney Research UK/British Renal Society - $£ 45,000$; Insulin Dependent Diabetes Trust - $£ 7,000$; SW Thames Kidney Fund - $£ 10,000$. Funding: others in last 5 years: Baxter Healthcare, Roche, Novartis.

$\mathrm{HG}^{1,6}$ Hugh Gallagher is an Honorary Senior Lecturer at St. George's and a renal physician at St. Helier Hospital with clinical responsibility for renal medicine in southwest Surrey. He is a panel member expert advisor for the QOF. He has received funding from several pharmaceutical companies for educational presentations on CKD, and an honorarium from a GP magazine to write an article on CKD (jointly with SdeL).

Received: 11 December 2010 Accepted: 5 August 2011

Published: 5 August 2011

\section{References}

1. Saxena S, Car J, Eldred D, Soljak M, Majeed A: Practice size, caseload, deprivation and quality of care of patients with coronary heart disease, hypertension and stroke in primary care: national cross-sectional study. BMC Health Serv Res 2007, 7:96.

2. Murray J, Saxena S, Millett C, Curcin V, de Lusignan S, Majeed A: Reductions in risk factors for secondary prevention of coronary heart disease by ethnic group in south-west London: 10-year longitudinal study (1998-2007). Fam Pract 2010.

3. Lusignan S, Sismanidis C, Carey IM, DeWilde S, Richards N, Cook DG: Trends in the prevalence and management of diagnosed type 2 diabetes 19942001 in England and Wales. BMC Fam Pract 2005, 22;6(1):13.

4. Gomez GB, de Lusignan S, Gallagher H: Chronic kidney disease: a new priority for primary care. Br J Gen Pract 2006, 56(533):908-10.

5. New JP, Middleton RJ, Klebe B, Farmer CK, de Lusignan S, Stevens PE, O'Donoghue DJ: Assessing the prevalence, monitoring and management of chronic kidney disease in patients with diabetes compared with those without diabetes in general practice. Diabet Med 2007, 24(4):364-9.

6. de Lusignan S, Chan T: The development of primary care information technology in the United kingdom. J Ambul Care Manage 2008, 31(3):201-10.

7. Cupples ME, Byrne MC, Smith SM, Leathem CS, Murphy AW: Secondary prevention of cardiovascular disease in different primary healthcare systems with and without pay-for-performance. Heart 2008, 94(12):1594-600.

8. Millett C, Bottle A, Ng A, Curcin V, Molokhia M, Saxena S, Majeed A: Pay for perfomance and the quality of diabetes management in individuals with and without co-morbid medical conditions. J R Soc Med 2009, 102(9):369-77.

9. Campbell SM, Reeves D, Kontopantelis E, Sibbald B, Roland M: Effects of pay for performance on the quality of primary care in England. N Engl J Med 2009, 361(4):368-78.

10. National Institute for Health and Clinical Excellence (NICE): Chronic kidney disease. National clinical guideline for early identification and management in adults in primary and secondary care (Clinical Guideline 73). London; NICE; 2008 [http://www.nice.org.uk/Guidance/CG73/Guidance/ $\mathrm{pdf} /$ English].

11. Clinical Knowledge Summaries (CKS): Indicators related to chronic kidney disease in the Quality and Outcomes Framework (QOF) of the General Medical Services (GMS) contract.[http://www.cks.nhs.uk/

chronic_kidney_disease_not_diabetic/management/ goals_and_outcome_measures/qof_indicators].

12. de Lusignan S, Gallagher H, Stevens P, Harris K, Dmetrieva O, Tahir A, Rafi I, Tomson C, O'Donoghue D: Chronic Kidney Disease: Frequently Asked Questions. London; BMA General Practitioners Committee \& NHS Employers; 32011 [http://www.nhsemployers.org/SiteCollectionDocuments/ Chronic kidney disease_FAQs\%20-\%20ja040711.pdf].

13. Gallagher H, de Lusignan S, Harris K, Cates C: Quality-improvement strategies for the management of hypertension in chronic kidney disease in primary care: a systematic review. Br J Gen Pract 2010, 60(575):258-65.

14. NHS The Information Centre. Statistics and data collections: The Quality and Outcomes Framework.[http://www.ic.nhs.uk/statistics-and-datacollections/audits-and-performance/the-quality-and-outcomes-framework].
15. Stevens PE, O'Donoghue DJ, de Lusignan S, Van Vlymen J, Klebe B, Middleton R, Hague N, New J, Farmer CK: Chronic kidney disease management in the United Kingdom: NEOERICA project results. Kidney Int 2007, 72(1):92-9.

16. Grol R: Successes and failures in the implementation of evidence-based guidelines for clinical practice. Med Care 2001, 39(8 Supp 12):|146-54.

17. Crinson I, Gallagher H, Thomas N, de Lusignan S: How ready is general practice to improve quality in chronic kidney disease? A diagnostic analysis. Br J Gen Pract 2010, 60(575):403-9.

18. de Lusignan S, Gallagher H, Chan T, Thomas N, van Vlymen J, Nation M, Jain N, Tahir A, du Bois E, Crinson I, Hague N, Reid F, Harris K: The QICKD study protocol: a cluster randomised trial to compare quality improvement interventions to lower systolic BP in chronic kidney disease (CKD) in primary care. Implement Sci 2009, 14;4:39.

19. Hulscher ME, Laurant MG: Grol RP. Process evaluation on quality improvement interventions. Qual Saf Health Care 2003, 12(1):40-6.

20. de Lusignan S: An educational intervention, involving feedback of routinely collected computer data, to improve cardiovascular disease management in UK primary care. Methods Inf Med 2007, 46(1):57-62.

21. de Lusignan S, Tomson C, Harris K, van Vlymen J, Gallagher H: Creatinine fluctuation has a greater effect than the formula to estimate glomerular filtration rate (eGFR) on the prevalence of chronic kidney disease (CKD). Accepted for publication: Nephron Clinical Practice; Manuscript No: 201003015

22. Bowling A: Research Methods in Health. Investigating health and health services. Maidenhead; Open University Press; "Third 2009.

23. de Lusignan S, Khunti K, Belsey J, Hattersley A, van Vlymen J, Gallagher H, Millett C, Hague NJ, Tomson C, Harris K, Majeed A: A method of identifying and correcting miscoding, misclassification and misdiagnosis in diabetes: a pilot and validation study of routinely collected data. Diabet Med 2010, 27(2):203-9.

24. van Vlymen J, de Lusignan S, Hague N, Chan T, Dzregah B: Ensuring the Quality of Aggregated General Practice Data: Lessons from the Primary Care Data Quality Programme (PCDQ). Stud Health Technol Inform 2005, 116:1010-5.

25. Israni RK, Shea JA, Joffe MM, Feldman HI: Physician characteristics and knowledge of CKD management. Am J Kidney Dis 2009, 54(2):238-47.

26. Agrawal V, Agarwal M, Ghosh AK, Barnes MA, McCullough PA: Identification and Management of Chronic Kidney Disease Complications by Internal Medicine Residents: A National Survey. Am J Ther 2009.

27. Wenghofer E, Klass D, Abrahamowicz M, Dauphinee D, Jacques A, Smee S, Blackmore D, Winslade N, Reidel K, Bartman I, Tamblyn R: Doctor scores on national qualifying examinations predict quality of care in future practice. Med Educ 2009, 43(12):1166-73.

28. Currin L, Waller G, Schmidt U: Primary care physicians' knowledge of and attitudes toward the eating disorders: do they affect clinical actions? Int J Eat Disord 2009, 42(5):453-8.

29. Ingram J: Multiprofessional training for breastfeeding management in primary care in the UK. Int Breastfeed J 2006, 28;1(1):9.

30. Fink P, Sørensen L, Engberg M, Holm M, Munk-Jørgensen P: Somatization in primary care. Prevalence, health care utilization, and general practitioner recognition. Psychosomatics 1999, 40(4):330-8.

31. Turner S, lliffe S, Downs M, Wilcock J, Bryans M, Levin E, Keady J, O'Carroll R: General practitioners' knowledge, confidence and attitudes in the diagnosis and management of dementia. Age Ageing 2004, 33(5):461-7.

32. Byrne M, Doherty S, McGee HM, Murphy AW: General practitioner views about discussing sexual issues with patients with coronary heart disease: a national survey in Ireland. BMC Fam Pract 2010, 11:40

33. Klebe B, Irving J, Stevens PE, O'Donoghue DJ, de Lusignan S, Cooley R, Hobbs H, Lamb EJ, John I, Middleton R, New J, Farmer CK: The cost of implementing UK guidelines for the management of chronic kidney disease. Nephrol Dial Transplant 2007, 22(9):2504-12.

\section{Pre-publication history}

The pre-publication history for this paper can be accessed here: http://www.biomedcentral.com/1471-2296/12/83/prepub

doi:10.1186/1471-2296-12-83

Cite this article as: Tahir et al:: Confidence and quality in managing CKD compared with other cardiovascular diseases and diabetes mellitus: a linked study of questionnaire and routine primary care data. BMC Family Practice 2011 12:83. 